\title{
Precise measurement of quantum observables with neural-network estimators
}

\author{
Giacomo Torlai $\odot,{ }^{1,}{ }^{*}$ Guglielmo Mazzola, ${ }^{2}$ Giuseppe Carleo, ${ }^{1}$ and Antonio Mezzacapo ${ }^{3}$ \\ ${ }^{1}$ Center for Computational Quantum Physics, Flatiron Institute, New York, New York 10010, USA \\ ${ }^{2}$ IBM Research Zurich, Saumerstrasse 4, 8803 Ruschlikon, Switzerland \\ ${ }^{3}$ IBM T.J. Watson Research Center, Yorktown Heights, New York 10598, USA
}

(Received 13 November 2019; revised manuscript received 28 February 2020; accepted 27 May 2020; published 17 June 2020)

\begin{abstract}
The measurement precision of modern quantum simulators is intrinsically constrained by the limited set of measurements that can be efficiently implemented on hardware. This fundamental limitation is particularly severe for quantum algorithms where complex quantum observables are to be precisely evaluated. To achieve precise estimates with current methods, prohibitively large amounts of sample statistics are required in experiments. Here, we propose to reduce the measurement overhead by integrating artificial neural networks with quantum simulation platforms. We show that unsupervised learning of single-qubit data allows the trained networks to accommodate measurements of complex observables, otherwise costly using traditional postprocessing techniques. The effectiveness of this hybrid measurement protocol is demonstrated for quantum chemistry Hamiltonians using both synthetic and experimental data. Neural-network estimators attain high-precision measurements with a drastic reduction in the amount of sample statistics, without requiring additional quantum resources.
\end{abstract}

DOI: 10.1103/PhysRevResearch.2.022060

\section{INTRODUCTION}

The measurement process in quantum mechanics has farreaching implications, ranging from the fundamental interpretation of quantum theory [1] to the design of quantum hardware [2]. The advent of medium-sized quantum computers has drawn attention to scalability issues different than control errors or decoherence, which nonetheless hinder the realization of complex quantum algorithms. Coherent and incoherent noise altering quantum states can be corrected in fault-tolerant architectures [3]. In contrast, the fluctuations introduced by a nonideal measurement protocol lead to intrinsic quantum noise which persists even in a fault-tolerant regime.

The most promising quantum computing platforms, such as superconducting or ion-trap processors, provide access to projective single-qubit nondemolition measurements $[4,5]$. Armed with these simple measurements, one is faced with a plethora of quantum simulation algorithms which rely on accurate estimations of specialized observables (e.g., ground and excited state energies, dipole moment, etc.). For practical purposes, in order to suppress the uncertainty arising from a suboptimal measurement apparatus, massive amounts of sample statistics need to be generated by the quantum device [6]. Complex estimators are then reconstructed through classical postprocessing of single-qubit data.

\footnotetext{
*gtorlai@flatironinstitute.org
}

Published by the American Physical Society under the terms of the Creative Commons Attribution 4.0 International license. Further distribution of this work must maintain attribution to the author(s) and the published article's title, journal citation, and DOI.
As the measurement precision remains tied to the interface between the quantum and the classical hardware, it becomes critical to develop methods capable of extracting more information from a given measurement dataset [7-12]. Given this, data-driven algorithms can provide a viable path towards improved accuracy and scalability in quantum simulation platforms.

Machine learning has recently shown its flexibility in finding approximate solutions to complex problems in a broad range of physics [13]. In particular, extensive theoretical work has demonstrated the potential of artificial neural networks in the context of quantum many-body physics [14-21]. The same approach has also been employed to enhance the capabilities of various quantum simulation platforms [22-27]. With the increasing stream of quantum data produced in laboratories, it is natural to expect further synergy between machine learning and experimental quantum hardware.

In this Rapid Communication, we propose to integrate neural networks with quantum simulators to increase the measurement precision of quantum observables. Using unsupervised learning on single-qubit data to learn approximately the quantum state underlying the hardware, neural networks can be deployed to generate estimators free of intrinsic quantum noise. This comes at the cost of a systematic bias from the imperfect quantum state reconstruction. We investigate the trade-off between these two sources of uncertainty for measurements of quantum chemistry Hamiltonians, costly with standard techniques [6]. We show a reduction of various orders of magnitude in the amount of data required to reach chemical accuracy for simulated data. For experimental data produced by a superconducting quantum hardware, we recover energy estimates with a low number of data points. This 
(a)

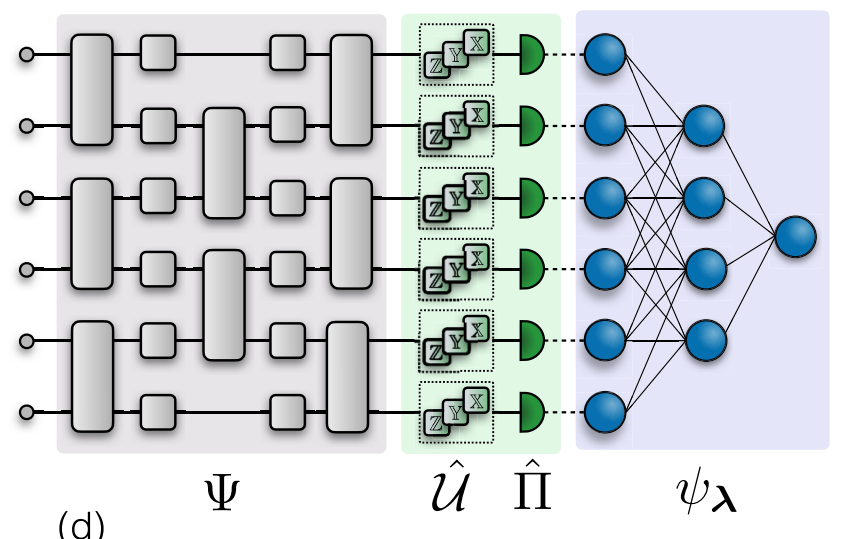

(d)

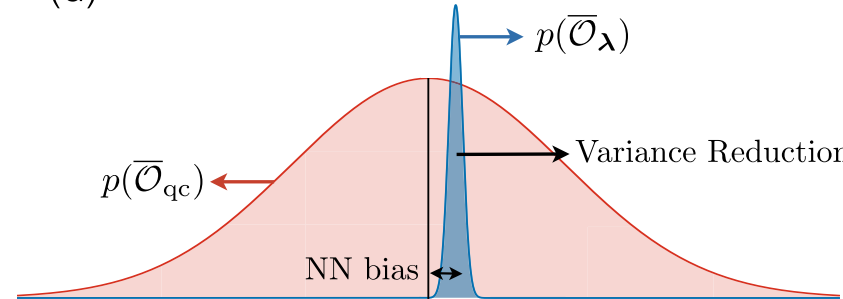

$\langle\hat{\mathcal{O}}\rangle$

FIG. 1. Measurements on quantum hardware with neuralnetwork estimators. (a) A quantum circuit prepares a quantum state $\Psi$. (b) Single-qubit measurements, consisting of a local rotation $\hat{\mathcal{U}}$ and a projective measurement $\hat{\Pi}$. (c) A neural network is trained on the output of the measuring apparatus to discover a representation $\psi_{\lambda}$ of the state $\Psi$ that retrieves the expectation value of a target quantum observable $\hat{\mathcal{O}}$. (d) The intrinsic measurement uncertainty is traded for a systematic reconstruction bias, leading to a measurement outcome distribution with lower variance.

opens up opportunities for quantum simulation on near-term quantum hardware [28].

\section{NEURAL-NETWORK ESTIMATORS}

We examine the task of estimating the expectation value of a generic observable $\hat{\mathcal{O}}$ on a quantum state $|\Psi\rangle$ prepared by a quantum computer with $N$ qubits. A direct measurement produces an estimator $\overline{\mathcal{O}} \approx\langle\hat{\mathcal{O}}\rangle$ with sample variance $\sigma^{2}[\mathcal{O}] \approx\left\langle\hat{\mathcal{O}}^{2}\right\rangle-\langle\hat{\mathcal{O}}\rangle^{2}$. This measurement is optimal when $|\Psi\rangle$ is the eigenstate of $\hat{\mathcal{O}}$ (i.e., $\sigma^{2}[\mathcal{O}]=0$ ), but requires sample statistics from the observable eigenbasis, typically not available on a quantum computer.

A more flexible measurement protocol can be devised by considering the expansion of the observable $\hat{\mathcal{O}}$ in terms of $K$ tensor products of Pauli operators

$$
\hat{\mathcal{O}}=\sum_{k=1}^{K} c_{k} \hat{P}_{k}, \quad \hat{P}_{k} \in\left\{\hat{\mathbb{1}}, \hat{\sigma}^{x}, \hat{\sigma}^{y}, \hat{\sigma}^{z}\right\}^{\otimes N},
$$

where $c_{k}$ are real coefficients. This decomposition allows one to estimate the expectation value from independent measurements of each Pauli operator, only requiring single-qubit data. In contrast to the direct measurement, the final estimator $\overline{\mathcal{O}}_{\mathrm{qc}}$ suffers an increased uncertainty $\epsilon_{\mathrm{qc}}=\sqrt{\sum_{k}\left|c_{k}\right|^{2} \sigma^{2}\left[P_{k}\right] / S}$, where $\sigma^{2}\left[P_{k}\right]$ is the sample variance of $\hat{P}_{k}$ and $S$ is the number of measurements (Supplemental Material [29]). The overhead in sample statistics to reduce this uncertainty becomes particularly severe for observables with a large number $K$ of Pauli operators.

We overcome this limitation by deploying unsupervised machine learning on single-qubit data to obtain an approximate reconstruction of the quantum state $|\Psi\rangle$ (Fig. 1). We call this reconstruction approximate in the sense that, unlike traditional quantum state tomography [30], we are primarily interested in the more restricted task of recovering measurement outcomes for the observable $\hat{\mathcal{O}}$. We first parametrize a generic many-body wave function by an artificial neural network. In a given reference basis $|\boldsymbol{\sigma}\rangle$ of the many-body Hilbert space (e.g., $|\boldsymbol{\sigma}\rangle=\left|\sigma_{1}^{z}, \ldots, \sigma_{N}^{z}\right\rangle, \sigma_{i}^{z}=\{0,1\}$ ), the neural network provides a parametric encoding of the amplitudes $\psi_{\lambda}(\sigma)=\left\langle\sigma \mid \psi_{\lambda}\right\rangle$ into a set of complex-valued weights $\lambda$ [16]. Specifically, we implement the restricted Boltzmann machine (RBM) [31], a physics-inspired generative neural network currently explored in many areas of condensed matter physics and quantum information $[32,33]$.

The quantum state reconstruction is carried out by training the neural network on a dataset $\mathcal{D}$ of $M$ single-qubit projective measurements, obtained from the target quantum state $|\Psi\rangle$ prepared by the hardware. ${ }^{1}$ Using an extension of unsupervised learning [20], the network parameters $\lambda$ are optimized via gradient descent to minimize the statistical distance between the probability distribution underlying the data and the RBM projective measurement probability. We adopt the standard measure given by the Kullbach-Leibler divergence

$$
\mathcal{C}_{\lambda}=-\frac{1}{M} \sum_{\sigma^{b} \in \mathcal{D}} \ln \left|\psi_{\lambda}\left(\sigma^{b}\right)\right|^{2},
$$

where $\boldsymbol{\sigma}^{\boldsymbol{b}}$ is an $N$-bit string $\left(\sigma_{1}^{b_{1}}, \ldots, \sigma_{N}^{b_{N}}\right)$ and $\boldsymbol{b}$ are Pauli bases $\left(b_{j}=\{x, y, z\}\right)$ drawn uniformly from the set of Pauli operators $\hat{P}_{k}$ appearing in Eq. (1) (Supplemental Material [29]). The reconstruction algorithm remains efficient as long as the number of nondiagonal Pauli matrices appearing in each single $\hat{P}_{k}$ increases subpolynomially with $N$.

Once the optimal parameters are selected according to cross-validation on held-out data, measurements of specialized observables can be performed by the neural network [17]. The expectation value of the quantum observable is simply approximated by the statistical estimator

$$
\overline{\mathcal{O}}_{\lambda}=\frac{1}{n_{\mathrm{MC}}} \sum_{j=1}^{n_{\mathrm{MC}}} \frac{\left\langle\boldsymbol{\sigma}_{j}|\hat{\mathcal{O}}| \psi_{\lambda}\right\rangle}{\left\langle\boldsymbol{\sigma}_{j} \mid \psi_{\lambda}\right\rangle},
$$

where $\left\{\sigma_{1}, \ldots, \sigma_{n_{\mathrm{MC}}}\right\}$ is a set of $n_{\mathrm{MC}}$ configurations drawn from the probability distribution $\left|\psi_{\lambda}(\sigma)\right|^{2}$ via Monte Carlo (MC) sampling. Here lies the critical advantage of the neuralnetwork estimator: despite that the wave function $\psi_{\lambda}(\sigma)$ is reconstructed from single-qubit data generated by the quantum computer, the measurement it produces is not affected by the intrinsic quantum noise. This is in fact equivalent to the direct

\footnotetext{
${ }^{1}$ Quantum state reconstruction was performed with the NetKet software [48].
} 


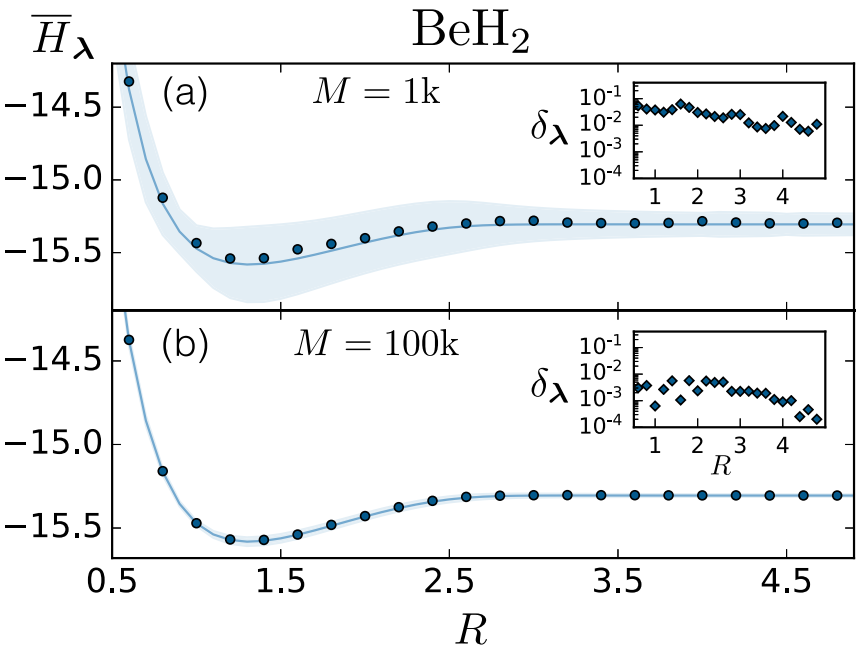

FIG. 2. Reconstruction of the potential energy surface of the $\mathrm{BeH}_{2}$ molecule (hartree and angstrom units). We show, for dataset sizes $M=10^{3}$ (a) and $M=10^{5}$ (b), the comparison between the exact ground-state energy $E_{0}$ (solid lines) and the energies obtained with the neural-network estimator (markers). The shaded regions span one standard deviation for the estimate on the quantum hardware with a standard averaging method using $M$ measurements. In the insets, we show the deviations $\delta_{\lambda}=\left|E_{0}-\bar{H}_{\lambda}\right|$ of the RBM estimators from the exact energies. measurement scheme where data is collected in the eigenbasis of the observable $\hat{\mathcal{O}}$ (Supplemental Material [29]).

\section{RESULTS}

The methods presented can be used to estimate a variety of observables in quantum simulations of many-body systems. Here, we specifically consider molecular energies in electronic structure calculations, using a second-quantized fermionic Hamiltonian $\hat{H}$. Other observables of interest may be the molecular dipole moments [34] or the excitation operators relevant in quantum subspace algorithms to compute excited state energies [35,36].

For these fermionic systems, the number of Pauli operators $K$ in Eq. (1) can grow up to the fourth power in the number of orbitals considered [37]. The resulting fast growth in measurement complexity remains a roadblock for quantum simulations on near-term hardware based on low depth quantum-classical hybrid algorithms, such as variational quantum eigensolvers [38].

We generate synthetic measurement datasets, sampling from the exact ground state of beryllium hydride $\left(\mathrm{BeH}_{2}\right)$, calculated by exact diagonalization of an $N=6$ qubit Hamiltonian. The latter is obtained from a second-quantized fermionic Hamiltonian in the atomic STO-3G basis through a parity transformation and qubit tapering from molecular symmetries [38,39]. We train a set of RBMs at different interatomic
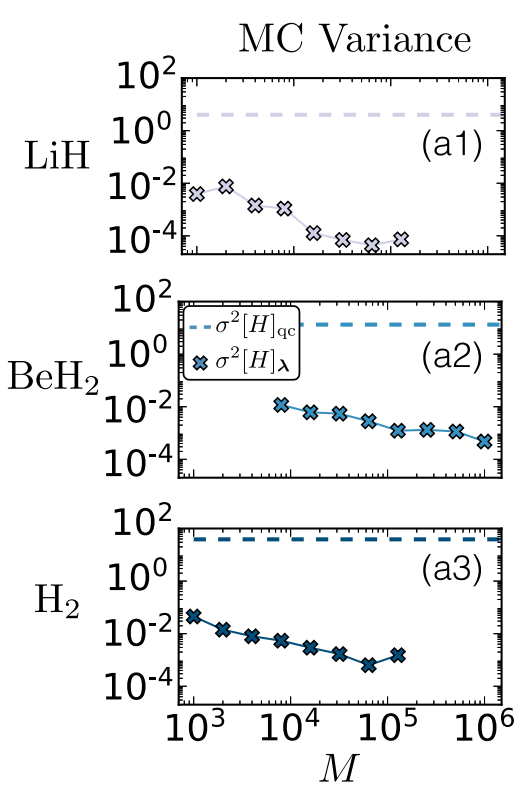

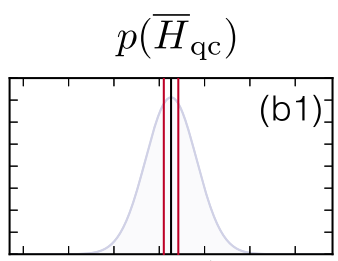

$-8.904$

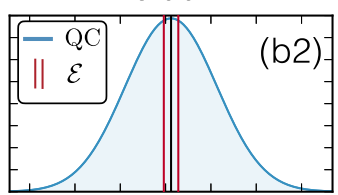

$-19.039$

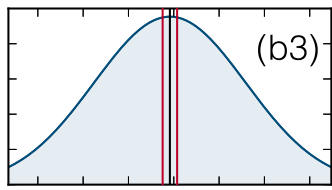

$-1.861$

$\bar{H}_{\mathrm{qc}}$
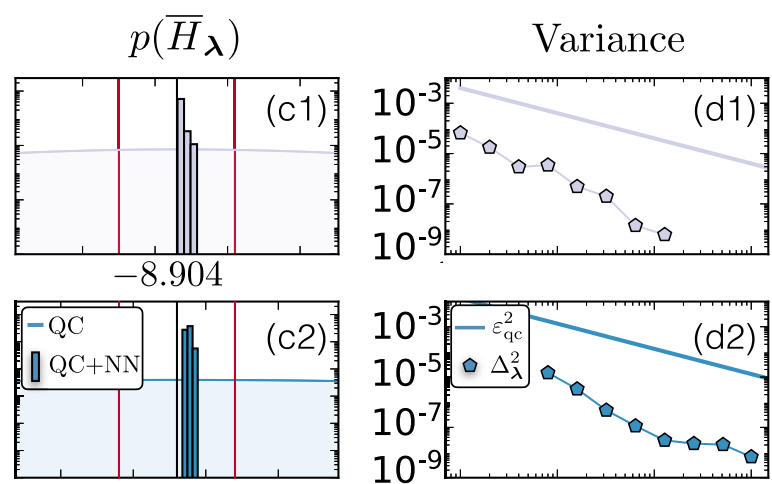

$-19.039$

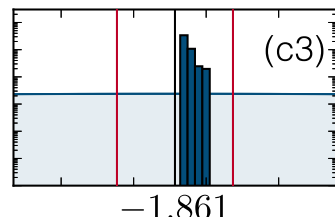

$\bar{H}_{\boldsymbol{\lambda}}$
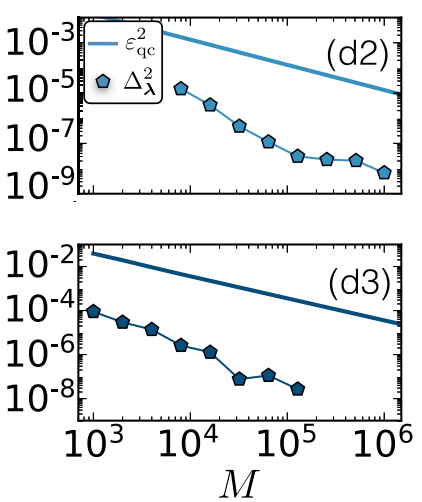

FIG. 3. Measurement uncertainty of neural-network estimators for molecular systems. The energy units are hartrees. (a1)-(a3) Statistical variance in the MC sampling of the neural network as a function of the size $M$ of the training dataset. We compare this with the variance $\sigma^{2}[H]_{\mathrm{qc}}=\sum_{k=1}^{K}\left|c_{k}\right|^{2} \sigma^{2}\left[P_{k}\right]$ arising from independent estimation of each Pauli operator with $S=M / K$ measurements. (b1)-(b3) Energy measurement distribution on the quantum computer. The red lines bound the chemical accuracy interval $\mathcal{E}=1.6 \times 10^{-3}$-the error in energy that changes a predicted chemical reaction rate by an order of magnitude at room temperature. The total number of measurements $M$ is set to 128000,1024 000, and 128000 for the $\mathrm{LiH}, \mathrm{BeH}_{2}$ (STO-3G basis), and $\mathrm{H}_{2}$ (6-31G basis) molecules, respectively. (c1)-(c3) Energy measurement distribution of the neural-network estimator (histogram), on the same number of measurements used in (b1)-(b3). All estimates fall within chemical accuracy from the true value $E_{0}$. (d1)-(d3) Energy errors induced by imperfect state reconstruction. We compare, for different dataset sizes $M$, the sample variance of the mean $\varepsilon_{\mathrm{qc}}^{2}=\sigma^{2}[H]_{\mathrm{qc}} / S$ with the variance of the distribution of the neural-network estimator $\Delta_{\lambda}^{2}$, calculated from the energy histograms in (c1)-(c3). 
separations $R$ using datasets $\mathcal{D}$ of increasing size $M$, and perform measurements of the molecular Hamiltonians $\bar{H}_{\lambda}{ }^{2}$ We show in Fig. 2 the neural-network estimators over the entire molecular energy surface. Comparing these measurements with exact energies shows that a relatively good precision can be achieved with as low as $M=10^{3}$ (total) measurements [Fig. 2(a)]. For a given number of measurements $M$, the neural-network estimator provides better estimates with respect to the conventional estimator $\bar{H}_{\mathrm{qc}}$.

The higher precision of estimators produced by the neural networks originates from the direct parametrization of the many-body wave function, eliminating any intrinsic quantum noise. In turn, the imperfect quantum reconstruction leads to two additional sources of uncertainty: a MC variance of statistical nature, and a systematic bias in the expectation value. In the following, we investigate these noise sources for the $\mathrm{BeH}_{2}$ molecule, as well as the lithium hydride $(\mathrm{LiH})$ in STO-3G basis and the hydrogen $\left(\mathrm{H}_{2}\right)$ molecule in the 6-31G basis, encoded in $N=4$ and $N=8$ qubits, respectively (we consider the geometry at the bond distance). We estimate the uncertainty of the measurement with the quantum computer using the exact variance calculated on the ground-state wave function, with $S=M / K$ measurements per Pauli operator. We point out that, while qubitwise commuting Pauli operators can be measured simultaneously, the resulting variance reduction is about a factor 2 for the molecules considered here [38].

The statistical uncertainty from the $\mathrm{MC}$ averaging is given by $\epsilon_{\lambda}=\sqrt{\sigma^{2}[H]_{\lambda} / n_{\mathrm{MC}}}$, where $\sigma^{2}[H]_{\lambda}$ is the variance of $\hat{H}$ on the samples generated by the neural network (Supplemental Material [29]). Since the target state (i.e., ground state) is an eigenstate of the observable, a perfect reconstruction would lead to zero variance. Deviations from the exact ground state set the amount $\sigma^{2}\left[H_{\lambda}\right]>0$ of statistical uncertainty in the sampling. In Figs. 3(a1)-3(a3), we show the MC variance for training datasets of increasing size $M$. Here, we fix the amount of MC samples to $n_{\mathrm{MC}}=10^{5}$, which is sufficient to make statistical fluctuations negligible. As expected, the MC variance decreases as $M$ grows larger and the quality of the reconstruction improves, with significant reduction compared to the variance $\sigma^{2}[H]_{\mathrm{qc}}$ obtained from standard postprocessing.

The reconstruction error in the neural-network estimator is also affected by finite-size deviations in the training dataset. To understand this contribution, we train a collection of 100 RBMs on independent measurement datasets, and compare the measurement distribution with the one obtained from standard averaging [Figs. 3(b1)-3(b3)]. By examining histograms of energies built from separate dataset realizations [Figs. 3(c1)-3(c3)], we observe that for sufficiently large $M$ the distribution of the neural-network estimator sharply peaks and gets close to the exact expectation value, with a positive offset due to the energy variational principle. For a quantitative comparison between the two distributions, we show in Figs. 3(d1)-3(d3) the variance of the mean for the neural-network estimator $\epsilon_{\lambda}^{2}$ (estimated from the histograms). We observe about two orders of magnitude improvement over the uncertainty $\epsilon_{\mathrm{qc}}^{2}$ of the standard estimator. Further systematic errors due to approximate representability have

\footnotetext{
${ }^{2}$ Molecular Hamiltonians were obtained with Qiskit Aqua [49].
}
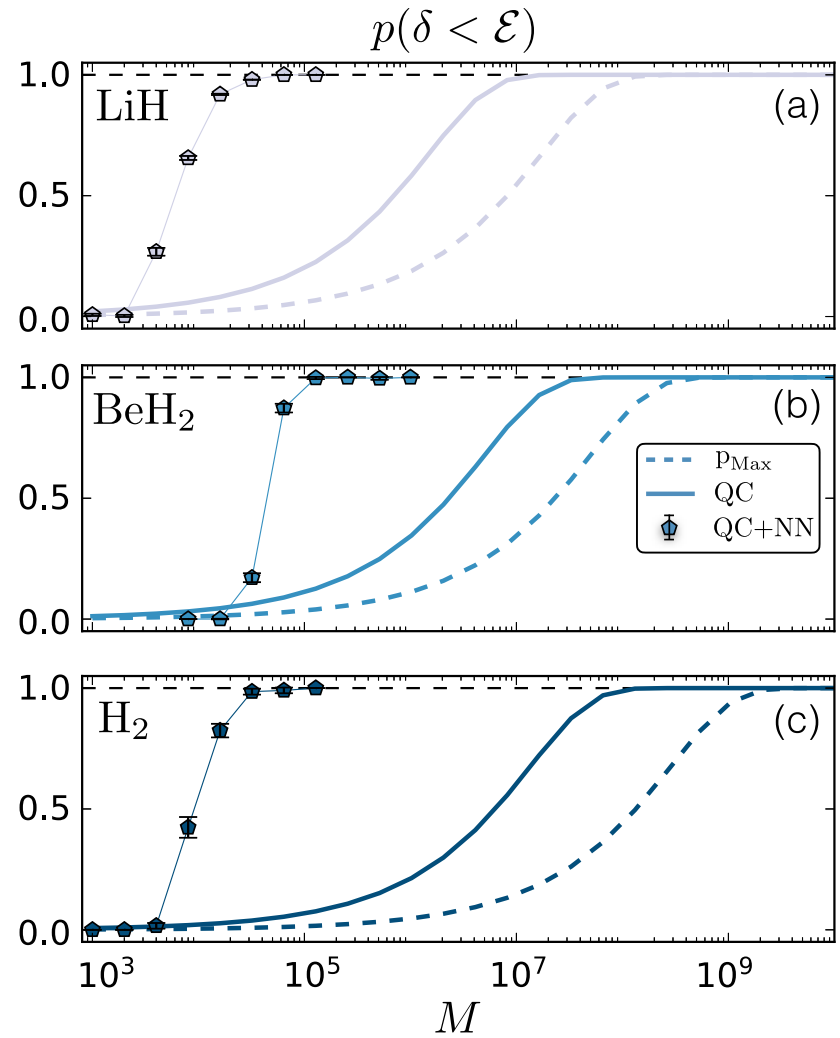

FIG. 4. Probability of reaching chemical accuracy as a function of the total number of measurements $M$ for $\mathrm{LiH}$ (a), $\mathrm{BeH}_{2}$ (b), and $\mathrm{H}_{2}(\mathrm{c})$. We compute the probability $p(\delta<\mathcal{E})$ to obtain a final energy estimate within chemical accuracy from the exact groundstate energy between. Plotted are probabilities for the neural-network estimator and the standard one. An upper bound $p_{\operatorname{Max}}$ to the latter is also shown, obtained by setting $\sigma^{2}[H]_{\mathrm{qc}}=\left(\sum_{k}\left|c_{k}\right|\right)^{2}$.

been shown to be negligible for molecular systems of larger sizes [40].

The total uncertainty in the final measurement estimator is a combination of systematic bias and statistical noise. We quantify the combined effect by considering the probability $p(\delta<\mathcal{E})$ that the deviation $\delta=\left|E_{0}-\bar{H}\right|$ from the ground-state energy $E_{0}$ is smaller than chemical accuracy $\mathcal{E}$. The specific value, which depends on thermal fluctuations at room temperature, is fixed to $\mathcal{E}=1.6 \times 10^{-3} \mathrm{Ha}$. A simple calculation leads to $p\left(\delta_{\mathrm{qc}}<\mathcal{E}\right)=\operatorname{erf}\left(\mathcal{E} \sqrt{S / 2 \sigma^{2}[H]_{\mathrm{qc}}}\right)$ for the standard estimator. We evaluate this probability for the neural-network estimator by independently resampling each neural-network across the separate training realizations. We show the results in Fig. 4, where we also include an upper bound $p_{\text {Max }}$ often referenced in literature [6,9]. We observe drastic improvements up to three orders of magnitude in the total measurements $M$ required to get to $p\left(\delta_{\lambda}<\mathcal{E}\right)=1$.

Finally, we show estimations of molecular energies with experimental data obtained in a variational quantum eigensolver. We use data from Ref. [41], which consist of samples from an approximate ground-state preparation of the $\mathrm{LiH}$ molecule on superconducting quantum hardware. In Fig. 5, we plot the energy profile reconstructed by the neural network, showing a good agreement using only a fraction of 


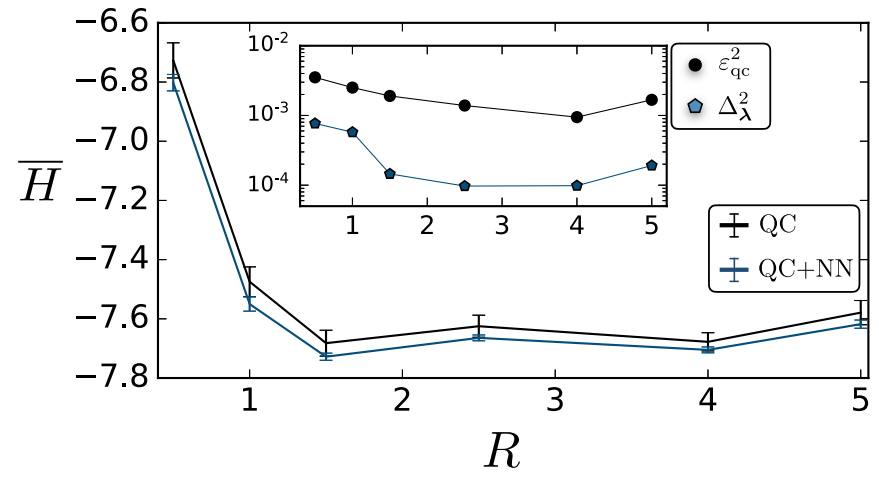

FIG. 5. Molecular energy (hartrees) of LiH from experimental data generated by a superconducting quantum processor [41], as a function of the interatomic distance (angstroms). The inset shows the variance $\varepsilon_{\mathrm{qc}}^{2}$ from subsampling $5 \times 10^{3}$ measurements out of $2.5 \times 10^{6}$ data points, and the corresponding variance $\Delta_{\lambda}^{2}$ obtained by separate neural-network reconstructions.

the total experimental measurements. Note that decoherence determines a discrepancy between the reconstructed and the measured profile, since our RBM makes a pure state assumption, which is not exactly verified experimentally.

To estimate the uncertainty, we train $50 \mathrm{RBMs}$ on separate datasets obtained by subsampling $M=5 \times 10^{3}$ measurement data points, out of the original $2.5 \times 10^{6}$ measurements in [41]. Despite the mixing in the quantum state underlying the measurements, the uncertainties in the neural-network estimators are systematically lower than the standard measurement scheme, similarly to what is observed in synthetic data.

\section{CONCLUSIONS}

We have introduced a procedure to measure complex observables in quantum hardware. The approach is based on approximate quantum state reconstruction tailored to retrieve a quantum observable of interest. For the particularly demanding case of quantum chemistry applications, we have provided evidence that neural-network estimators achieve precise measurements with a reduced amount of sample statistics.

An intriguing open question for future research is the systematic understanding of machine learning-based quantum state reconstruction. For positive wave functions, a favorable asymptotic reconstruction scaling has been recently shown [42]. For nonpositive states, such as ground states of interacting electrons, recent works addressed representability $[40,43,44]$, while much less is known for the reconstruction complexity, leaving open prospects for future studies.

For measurement data generated by the experimental hardware, we have also assumed that the quantum state is approximately pure. When decoherence effects substantially corrupt the state, density-matrix neural-network reconstruction techniques $[45,46]$ could be employed as an alternative to the algorithm presented here. Generative models other than neural networks [47] could also be explored in this setup.

Finally, the increased measurement precision with lower sample complexity makes neural-network estimators a powerful asset in variational quantum simulations of ground states by hybrid algorithms using low-depth quantum circuits $[38,50]$. It is natural to expect integration of generative models in the feedback loop for the quantum circuit optimization. With the ever-increasing size of quantum hardware, we envision that machine learning will play a fundamental role in the development of the next generation of quantum technologies.

\section{ACKNOWLEDGMENTS}

We thank J. Carrasquilla, M. T. Fishman, J. Gambetta, and R. G. Melko for useful discussions. We thank A. Kandala for the availability of raw experimental data from Ref. [41]. The Flatiron Institute is supported by the Simons Foundation. A.M. acknowledges support from the IBM Research Frontiers Institute. Numerical simulations have been carried out on the Simons Foundation Supercomputing Center.
[1] M. Schlosshauer, J. Kofler, and A. Zeilinger, Stud. Hist. Philos. Sci., Part B: Stud. Hist. Philosoph. Mod. Phys. 44, 222 (2013).

[2] A. A. Clerk, M. H. Devoret, S. M. Girvin, F. Marquardt, and R. J. Schoelkopf, Rev. Mod. Phys. 82, 1155 (2010).

[3] E. T. Campbell, B. M. Terhal, and C. Vuillot, Nature (London) 549, 172 (2017).

[4] A. Wallraff, D. I. Schuster, A. Blais, L. Frunzio, R. S. Huang, J. Majer, S. Kumar, S. M. Girvin, and R. J. Schoelkopf, Nature (London) 431, 162 (2004).

[5] D. J. Wineland, J. C. Bergquist, W. M. Itano, and R. E. Drullinger, Opt. Lett. 5, 245 (1980).

[6] D. Wecker, M. B. Hastings, and M. Troyer, Phys. Rev. A 92, 042303 (2015).

[7] A. Jena, S. Genin, and M. Mosca, arXiv:1907.07859.

[8] T.-C. Yen, V. Verteletskyi, and A. F. Izmaylov, J. Chem. Theory Comput. 16, 2400 (2020).

[9] W. J. Huggins, J. McClean, N. Rubin, Z. Jiang, N. Wiebe, K. B. Whaley, and R. Babbush, arXiv:1907.13117.
[10] P. Gokhale, O. Angiuli, Y. Ding, K. Gui, T. Tomesh, M. Suchara, M. Martonosi, and F. T. Chong, arXiv:1907.13623.

[11] O. Crawford, B. van Straaten, D. Wang, T. Parks, E. Campbell, and S. Brierley, arXiv:1908.06942.

[12] A. Zhao, A. Tranter, W. M. Kirby, S. F. Ung, A. Miyake, and P. Love, arXiv:1908.08067.

[13] G. Carleo, I. Cirac, K. Cranmer, L. Daudet, M. Schuld, N. Tishby, L. Vogt-Maranto, and L. Zdeborová, Rev. Mod. Phys. 91, 045002 (2019).

[14] J. Carrasquilla and R. G. Melko, Nat. Phys. 13, 431 (2017).

[15] L. Wang, Phys. Rev. B 94, 195105 (2016).

[16] G. Carleo and M. Troyer, Science 355, 602 (2017).

[17] G. Torlai and R. G. Melko, Phys. Rev. B 94, 165134 (2016).

[18] E. P. L. van Nieuwenburg, Y.-H. Liu, and S. D. Huber, Nat. Phys. 13, 435 (2017).

[19] M. Koch-Janusz and Z. Ringel, Nat. Phys. 14, 578 (2018). 
[20] G. Torlai, G. Mazzola, J. Carrasquilla, M. Troyer, R. Melko, and G. Carleo, Nat. Phys. 14, 447 (2018).

[21] M. Bukov, A. G. R. Day, D. Sels, P. Weinberg, A. Polkovnikov, and P. Mehta, Phys. Rev. X 8, 031086 (2018).

[22] A. Seif, K. A. Landsman, N. M. Linke, C. Figgatt, C. Monroe, and M. Hafezi, J. Phys. B: At., Mol. Opt. Phys. 51, 174006 (2018).

[23] B. S. Rem, N. Käming, M. Tarnowski, L. Asteria, N. Fläschner, C. Becker, K. Sengstock, and C. Weitenberg, Nat. Phys. 15, 917 (2019).

[24] A. Bohrdt, C. S. Chiu, G. Ji, M. Xu, D. Greif, M. Greiner, E. Demler, F. Grusdt, and M. Knap, Nat. Phys. 15, 921 (2019).

[25] G. Torlai, B. Timar, E. P. L. van Nieuwenburg, H. Levine, A. Omran, A. Keesling, H. Bernien, M. Greiner, V. Vuletić, M. D. Lukin, R. G. Melko, and M. Endres, Phys. Rev. Lett. 123, 230504 (2019).

[26] Y. Zhang, A. Mesaros, K. Fujita, S. D. Edkins, M. H. Hamidian, K. Ch'ng, H. Eisaki, S. Uchida, J. C. S. Davis, E. Khatami, and E.-A. Kim, Nature (London) 570, 484 (2019).

[27] Y. H. Teoh, M. Drygala, R. G. Melko, and R. Islam, Quantum Sci. Technol. 5, 024001 (2020).

[28] J. Preskill, Quantum 2, 79 (2018).

[29] See Supplemental Material at http://link.aps.org/supplemental/ 10.1103/PhysRevResearch.2.022060 for details on the measurement procedure in quantum hardware and the training of the neural-network wave functions.

[30] K. Banaszek, M. Cramer, and D. Gross, New J. Phys. 15, 125020 (2013).

[31] D. H. Ackley, G. E. Hinton, and T. J. Sejnowski, Cogn. Sci. 9, 147 (1985).

[32] G. Torlai and R. G. Melko, Ann. Rev. Condens. Matter Phys. 11, 325 (2020).

[33] R. G. Melko, G. Carleo, J. Carrasquilla, and J. I. Cirac, Nat. Phys. 15, 887 (2019).

[34] J. E. Rice, T. P. Gujarati, T. Y. Takeshita, J. Latone, M. Motta, A. Hintennach, and J. M. Garcia, arXiv:2001.01120.

[35] J. I. Colless, V. V. Ramasesh, D. Dahlen, M. S. Blok, M. E. Kimchi-Schwartz, J. R. McClean, J. Carter, W. A. de Jong, and I. Siddiqi, Phys. Rev. X 8, 011021 (2018).
[36] P. J. Ollitrault, A. Kandala, C.-F. Chen, P. K. Barkoutsos, A. Mezzacapo, M. Pistoia, S. Sheldon, S. Woerner, J. Gambetta, and I. Tavernelli, arXiv:1910.12890.

[37] D. Poulin, M. B. Hastings, D. Wecker, N. Wiebe, A. C. Doberty, and M. Troyer, Quantum Inf. Comput. 15, 361 (2015).

[38] A. Kandala, A. Mezzacapo, K. Temme, M. Takita, M. Brink, J. M. Chow, and J. M. Gambetta, Nature (London) 549, 242 (2017).

[39] S. Bravyi, J. M. Gambetta, A. Mezzacapo, and K. Temme, arXiv:1701.08213.

[40] K. Choo, A. Mezzacapo, and G. Carleo, Nat. Commun. 11, 2368 (2020).

[41] A. Kandala, K. Temme, A. D. Córcoles, A. Mezzacapo, J. M. Chow, and J. M. Gambetta, Nature (London) 567, 491 (2019).

[42] D. Sehayek, A. Golubeva, M. S. Albergo, B. Kulchytskyy, G. Torlai, and R. G. Melko, Phys. Rev. B 100, 195125 (2019).

[43] D. Luo and B. K. Clark, Phys. Rev. Lett. 122, 226401 (2019).

[44] D. Pfau, J. S. Spencer, A. G. de G. Matthews, and W. M. C. Foulkes, arXiv:1909.02487.

[45] G. Torlai and R. G. Melko, Phys. Rev. Lett. 120, 240503 (2018).

[46] J. Carrasquilla, G. Torlai, R. G. Melko, and L. Aolita, Nat. Mach. Intell. 1, 155 (2019).

[47] I. Glasser, R. Sweke, N. Pancotti, J. Eisert, and J. I. Cirac, arXiv:1907.03741.

[48] G. Carleo, K. Choo, D. Hofmann, J. E. T. Smith, T. Westerhout, F. Alet, E. J. Davis, S. Efthymiou, I. Glasser, S.-H. Lin, M. Mauri, G. Mazzola, C. B. Mendl, E. van Nieuwenburg, O. O'Reilly, H. Théveniaut, G. Torlai, F. Vicentini, and A. Wietek, SoftwareX 10, 100311 (2019).

[49] H. Abraham, I. Y. Akhalwaya, G. Aleksandrowicz, T. Alexander, G. Alexandrowics, E. Arbel, A. Asfaw, C. Azaustre, P. Barkoutsos, G. Barron et al., Qiskit: An open-source framework for quantum computing, 2019.

[50] C. Kokail, C. Maier, R. van Bijnen, T. Brydges, M. K. Joshi, P. Jurcevic, C. A. Muschik, P. Silvi, R. Blatt, C. F. Roos, and P. Zoller, Nature (London) 569, 355 (2019). 